\title{
Oncology protocols: how can we do better?
}

\author{
Sue C. Kaste
}

Received: 24 November 2010 /Revised: 21 December 2010 /Accepted: 4 January 2011 /Published online: 16 February 2011

(C) Springer-Verlag 2011

\begin{abstract}
Diagnostic imaging provides information that is critical to the clinical care of pediatric oncology patients. Such information is also vital for assessing the effects of treatment on specific tumors during clinical trials. The design of such trials mandates standardization of imaging techniques, tumor measurements, data analysis, consistency of data collection and image interpretation in order to make scientific decisions about treatment regimens. In addition to these considerations, the optimal imaging modality to answer the scientific questions and simultaneously provide excellent patient care must be determined while minimizing patient exposure to ionizing radiation. This presentation will discuss these factors and potential means of improving upon inconsistencies, and consider factors that might influence the future.
\end{abstract}

Keywords Childhood cancer Pediatric oncology

\section{Introduction}

Childhood cancer is the No. 1 cause of death from disease in children younger than 15 years of age. In the United States from 2002 to 2006, the number of affected people was estimated to be 179 cases per million [1], nearly 200,000 cases in children and adolescents younger than 20 years [2]. Innovative therapies, detailed surveillance and

Disclaimer The supplement this article is part of is not sponsored by the industry. Dr. Kaste has no financial interests, investigational or offlabel uses to disclose.

\section{S. C. Kaste $(\square)$}

St. Jude Children's Research Hospital,

MSN \#220, 262 Danny Thomas Place,

Memphis, TN 38105-3678, USA

e-mail: Sue.kaste@stjude.org improved assessment of tumor response to treatment have resulted in a steadily growing population at risk for late toxicities that can adversely affect not only quality but length of life. In the United States alone, this cohort is estimated to exceed 300,000 individuals [3].

As pediatric radiologists, we must develop the most sensitive, specific and innovative methods of anatomical and functional imaging for diagnosing tumor extent including metastatic disease, determining tumor effects on adjacent organs, tumor response to therapy, identification of tumor recurrence and the development of second malignant neoplasms. Further, we need to stay abreast of new diseasespecific, patient-specific and risk-adapted protocols in designing imaging surveillance.

All of this must be considered in the context of optimizing cure, identifying and minimizing adverse toxicities, growing survivorship, and late effects, and it must adhere to the dictum of "as low as reasonably achievable" (ALARA). With these factors and responsibilities in mind, we will consider methods and techniques directed at optimizing imaging data while minimizing risk and providing best clinical care.

\section{Limiting patient exposure to ionizing radiation}

Children undergoing therapy for childhood cancer can be exposed to excessive ionizing radiation merely by the number of diagnostic imaging studies to which they are subjected, most notably repeated CT scans and the evolving use of PET and PET-CT. We are well aware that children are more sensitive to ionizing radiation than adults [4]. The risk associated with exposure to ionizing radiation from medical imaging in the general pediatric population is compounded by the notable fact that childhood cancer 
survivors bear an additional 6-fold increased risk of developing a second cancer when compared to the general population. For those children who also received radiotherapy for their cancers, the 5-year risk for an additional cancer rises to 8 -fold increased risk [5, 6]. Further, because of their genetic makeup, a selected pediatric population is further predisposed to cancer-related syndromes and to second malignancies. Such cancer predisposition syndromes, though rare, include Li-Fraumeni, retinoblastoma, neurofibromatosis type 1 and basal cell carcinoma syndrome [5, 7-10].

We are responsible for minimizing radiation, developing techniques exploring and expanding the use of US and MR imaging, and in cases when exposure to ionizing irradiation is the ideal method, providing the most information while stringently adhering to ALARA principles.

\section{Imaging in clinical trials}

To choose the effective modality- and design-appropriate imaging protocols, imagers must understand that clinical trials are performed in stages, with each stage having a specific purpose [11]; imaging must be designed in this context.

Imaging in phase I and phase II clinical trials

Phase I and phase II clinical trials represent translation from preclinical (animal) trials into human populations. Imaging needs in such trials are unique, as a new therapeutic agent or regimen is the variable to be assessed [12] with determination of tolerated dose levels and toxicities. Thus, knowledge of the mechanisms of action of new drugs on both normal and cancer cells and the anticipated imaging findings are required to select the imaging modality and technique to answer the scientific questions. New multi-modality and multi-drug therapy prompt drug-related imaging for toxicity. One such example is the incorporation of anti-angiogenesis factors and VEGF agents into phase I and phase II clinical trials. Translation of preclinical findings of growth abnormalities in pediatric mice prompts imaging for such potential toxicity in pediatric patients [13].

Imaging in phase III and phase IV clinical trials

Phase III and phase IV clinical trials investigate the efficacy of treatment protocols with standard therapy and further evaluate safety and side effects. Tumor control is typically compared with prior regimens. Historical imaging findings need to be considered and translation of information between prior studies and optimal imaging protocols for new agents must be addressed. Further, the interpretation of protocol-driven imaging studies must be standardized. Central review of such cases strengthens the standardization of data collection and, hence, provides important insight into treatment response.

\section{Surveillance or off-therapy imaging}

End of therapy does not equate to end of care. Monitoring for recurrent disease and for development of second malignancies is ongoing for several years after treatment completion $[6,14,15]$. These regimens are typically tailored to salvage rates of relapse disease but vary widely among institutions and cooperative groups. One such example is the European use of US for monitoring Wilms tumor while the North American preference has historically been cross-sectional imaging.

Imaging for adverse oncotherapeutic sequelae is also warranted [16]. Monitoring varies widely with primary diagnosis, patient demographics at diagnosis (such as age, gender, race, genetic risk) and treatment exposures. One example is monitoring for development of osteonecrosis $(\mathrm{ON})$. ON is recognized worldwide as one of the most common toxicities related to the treatment of acute lymphoblastic leukemia (ALL) [17-20]. The prevalence and severity of detected involvement varies widely and depends upon imaging prompted by clinical symptoms or imaging sought regardless of symptoms and between radiographic and MR monitoring [21].

\section{Outcomes analysis of diagnostic imaging studies}

Technological advancement typically exceeds the ability to fully assess its utility, roles and limitations. It is not at all uncommon for a new modality to be incorporated into clinical trials and to become a relative standard of care for imaging in addition to multiple pre-existing modalities. One example is continued use of chest radiography when chest $\mathrm{CT}$ for assessment and detection of metastatic disease is also included in the protocol. Though chest radiography bears a relatively low exposure dose, it is an additional exposure compounding a patient's cumulative exposure to ionizing radiation. Few modality-specific outcomes publications are available. It falls within our responsibility as imagers and collaborators in study designs to engage the oncological community and direct assessment of the sensitivity and specificity of a given modality, its frequency of use and impact on tumor response assessments. Only through such analyses can we minimize radiation exposures, optimize disease detection and monitoring, and impact resource utilization. 


\section{Standardized imaging}

Standardization of imaging techniques is necessary to fully evaluate clinical questions. However, few imaging questions have historically been included in pediatric oncological studies. Factors that contribute to this deficit include limited knowledge of treatment study designs, varied access to imaging modalities among participating institutions, varied skills possessed by radiologists interpreting such studies, personal preferences of assessments by clinicians and radiologists, and cost and resource limitations. In multiinstitutional clinical trials, latitude in the imaging modality and techniques is tolerated in order to enroll adequate numbers of patients to answer the clinical questions. This latitude jeopardizes potentially important information that could be obtained from analyzing standardized imaging data.

Historically, imaging reviews have been performed in central locations, monitored by quality assurance specialists to minimize variations in tumor assessments and to assure accurate completion of data collection forms. Such reviews mandate reviewer travel, time away from work and sacrifice of weekends and vacation time, added costs related to travel, and review of numerous cases in a short period of time. With the evolution of electronic technology, "e-centralized" imaging review is now possible, obviating the need for time away, decreasing costs related to travel and permitting interdigitation of protocol-directed case reviews among clinical demands. Functions such as electronic transfer of images, multiinstitutional access and compatibility, accurate data collection and ease of use while assuring patient anonymity are challenges we currently face with growing this process.

As treatment protocols and endpoint assessments are standardized, so must be the imaging techniques and methods of imaging data capture. Variability in clinical imaging interpretations and report structures is well-known and generally accepted for clinical care. However, the value of standardized reporting is exemplified by endorsement and development by the Radiologic Society of North America (RSNA) [22] and the American College of Radiology (ACR) [23]. Similarly, data capture for hypothesis-driven studies requires even further refinement. Such details are critical to assessing disease response and include modality to determine tumor measurements, plane(s) in which measurements are determined, method of measurement, boundaries of tumor measurements and sequences used.

\section{Communication and collaborative efforts}

It is through communication with clinical oncologists, basic science researchers and oncological imagers that appropriate imaging can be designed and executed. The understanding of individual disease entities and expected responses to therapy results in appropriate design of imaging protocols and their interpretations.

\section{Conclusion}

The population of pediatric cancer survivors will continue to grow with continued treatment successes. We are at the crossroads of needing to be cognitive of imaging needs during treatment and limiting adverse events that can compromise quality and length of survivors' lives. Through ongoing collaborative efforts with clinical and basic science researchers we can propagate successes and contribute to study designs while minimizing adverse events.

\section{References}

1. U.S. Cancer Statistics Working Group (2010) United States Cancer Statistics: 1999-2007 Incidence and Mortality Web-based Report. Available via http://apps.nccd.cdc.gov/uscs/childhoodcancerdetailed byICCC.aspx. Accessed 14 Dec 2010

2. National Cancer Institute (2007) SEER Cancer Statistics Review, 1975-2007. Available via http://seer.cancer.gov/cgi-bin/csr/ $19752007 /$ search.pl?sort_order $=\&$ search_site $=\mathrm{c} 27 \&$ search 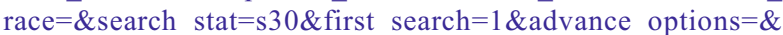 search_stat_list $=\mathrm{s} 30 \&$ search_site_list $=\mathrm{c} 27 \&$ search_race list $=$. Accessed 14 Dec 2010

3. Mariotto AB, Rowland JH, Yabroff KR et al (2009) Long-term survivors of childhood cancers in the United States. Cancer Epidemiol Biomark Prev 18:1033-1040

4. Hall EJ (2002) Introduction to session I: helical CT and cancer risk. Pediatr Radiol 32:225-227

5. Kleinerman RA (2009) Radiation-sensitive genetically susceptible pediatric sub-populations. Pediatr Radiol 39(Suppl 1):S27-S31

6. Inskip PD, Curtis RE (2007) New malignancies following childhood cancer in the United States, 1973-2002. Int J Cancer 121:2233-2240

7. Bhatia S, Sklar C (2002) Second cancers in survivors of childhood cancer. Nat Rev Cancer 2:124-132

8. Meadows AT (2005) A mouse model for studying therapyinduced cancers. Cancer Cell 8:271-273

9. Strahm B, Malkin D (2006) Hereditary cancer predisposition in children: genetic basis and clinical implications. Int $\mathrm{J}$ Cancer 119:2001-2006

10. Evans DG, Ramsden RT, Shenton A et al (2007) Mosaicism in neurofibromatosis type 2: an update of risk based on uni/ bilaterality of vestibular schwannoma at presentation and sensitive mutation analysis including multiple ligation-dependent probe amplification. J Med Genet 44:424-428

11. National Institutes of Health (NIH) (2010) Understanding clinical trials. Available via http://clinicaltrials.gov/ct2/info/understand. Accessed 16 Nov 2010

12. Reaman GH (2009) What, why, and when we image: considerations for diagnostic imaging and clinical research in the Children's Oncology Group. Pediatr Radiol 39(Suppl 1):S42-S45

13. Kimura H, Ng JM, Curran T (2008) Transient inhibition of the Hedgehog pathway in young mice causes permanent defects in bone structure. Cancer Cell 13:249-260

14. Bhatia S, Yasui Y, Robison LL et al (2003) High risk of subsequent neoplasms continues with extended follow-up of 
childhood Hodgkin's disease: report from the Late Effects Study Group. J Clin Oncol 21:4386-4394

15. Bhatti P, Veiga LH, Ronckers CX et al (2010) Risk of second primary thyroid cancer after radiotherapy for a childhood cancer in a large cohort study: an update from the childhood cancer survivor study. Radiat Res 174:741-752

16. Meadows AT (2003) Pediatric cancer survivors: past history and future challenges. Curr Probl Cancer 27:112-126

17. Karimova EJ, Rai SN, Ingle D et al (2006) MRI of knee osteonecrosis in children with leukemia and lymphoma: Part 2, clinical and imaging patterns. AJR 186:477-482

18. Mattano LA Jr, Sather HN, Trigg ME et al (2000) Osteonecrosis as a complication of treating acute lymphoblastic leukemia in children: a report from the Children's Cancer Group. J Clin Oncol 18:3262-3272
19. Sala A, Mattano LA Jr, Barr RD (2007) Osteonecrosis in children and adolescents with cancer-an adverse effect of systemic therapy. Eur J Cancer 43:683-689

20. Barr RD, Sala A (2008) Osteonecrosis in children and adolescents with cancer. Pediatr Blood Cancer 50:483-485

21. Kaste SC (2008) Skeletal toxicities of treatment in children with cancer. Pediatr Blood Cancer 50:469-473

22. (2010) RSNA Radiology Reporting Initiative. Available via http:// reportingwiki.rsna.org/index.php?title=Main_Page. Accessed 14 Dec 2010

23. (2010) ACR practice guideline for communication of diagnostic imaging findings. Available via http://www.acr.org/SecondaryMain MenuCategories/quality_safety/guidelines/dx/comm_diag_rad.aspx. Accessed 14 Dec 2010 